\section{A Simple Method of Photographing Ouchterlony Plates}

\section{J. H. JONES AND R. J. MARSHALL}

From the Institute of Pathology and the Department of Medical Photography, Royal Infirmary, Cardiff

The Ouchterlony technique of demonstrating precipitin bands in an agar gel (Ouchterlony, 1948) is one which is now widely used for diagnostic purposes. The difficulties involved in making a permanent accurate record of the results, an essential part of the technique, are indicated by the variety of photographic methods that have been used, the majority of which are time-consuming and involve the use of special photographic equipment.

We have been using a modification of a method first described by Bartlett (quoted by Gibson, 1954) which is accurate, rapid, and requires a minimum of equipment. This technique consists essentially of making a photogram of the agar plate on a lantern plate. Because of its simplicity it is of value to those using the gel-diffusion technique.

\section{Material and Methods}

Agar Plates.-The 1\% agar used is prepared according to the method of Feinberg (1956). The petri dishes are selected free from scratches and other defects in the glass. Before photographing it is an advantage to wash the surface of the agar several times with cold water to free it of dust particles. The surface of the agar is then covered with a layer of water which serves both to fill the cups, thus making them less prominent on the finished photograph, and to obliterate any surface defects of the agar.

Photography.-The lantern plates used are Ilford "special contrasty" grade. One is placed under the petri dish and exposed to light from a condenser enlarger. Bartlett's original method specified the use of a cold-cathode enlarger, though any vertical enlarger may be used; in fact, any point source of light is satisfactory. The illumination provided by an ordinary 60-watt pearl electric light bulb enclosed in a metal can having a $\frac{1}{4}$ in. diameter circular hole on its base has been used here. The lamp should be placed several feet above the precise centre of the plate.

Exposures, using an ordinary enlarger with its lens stopped down to an aperture of $f / 22$ or a screened pearl bulb, are in the range of seven to 10 seconds. Processing should be in accordance with the manufacturer's instructions.

This method produces a very satisfactory negative recording faithfully all the bands in the agar plate. From this contact prints or enlargements may be made. An even simpler method is to make the photogram direct on to contrasty bromide printing paper (Ilford grade 3 or 4) though, of course, a negative image of the bands is produced, but this appears to be quite satisfactory for routine purposes.

\section{REFERENCES}

Feinberg, J. G. (1956). Nature (Lond.), 178, 1406.

Gibson, H. L. (1954). Med. Radiogr. Photogr., $30,50$.

Ouchterlony, Ö. (1948). Acta path. microbiol. scand., 25, 186.


FIG. 1.-Positive and negative photograms of an Ouchterlony plate taken with a shielded pearl bulb and showing a variety of precipitin bands. The central cup contains rat serum and the peripheral cups contain sera from rabbits which had been immunized against various fractions of rat serum. 